\title{
Effect of Initial Stress and Magnetic Field on Propagation of Shear Wave in Non Homogeneous Anisotropic Medium Under Gravity Field
}

\author{
A.M. Abd-Alla ${ }^{\mathrm{a}}$, S.R. Mahmoud*,\#, and M.I.R. Helmi ${ }^{\mathrm{a}}$ \\ ${ }^{a}$ Mathematics Department, Faculty of Science, Taif University, K.S.A., Suadia Arab \\ ${ }^{b}$ Mathematics Department, J.T.C., King Abdul Aziz University, K.S.A., Suadia Arab
}

\begin{abstract}
In this paper, propagation of shear waves in a non-homogeneous anisotropic incompressible, magnetic field, gravity field and initially stressed medium has been studied. Analytical analysis reveals that the velocity of propagation of the shear waves depends upon the direction of propagation, the anisotropy, magnetic field, gravity field, non-homogeneity of the medium, and the initial stress. The frequency equation that determines the velocity of the shear wave has been obtained. The dispersion equations have been obtained and investigated for different cases. In fact, these equations are an agreement with the corresponding classical results when the medium is isotropic. The results have been obtained are discussed and presented graphically.
\end{abstract}

Keywords: Incompressible, initial-stress, anisotropic, shear-wave, gravity field, magnetic field.

\section{INTRODUCTION}

In the recent years, more attention has given to the usage of the anisotropic material in engineering applications in considerable research activity. Problem of shear waves in an orthotropic elastic medium is been very important for the possibility of its extensive application in various branches of Science and Technology, particularly in Optics, Earthquake science, Acoustics, Geophysics and Plasma physics.

Shear waves propagating over the surface of homogeneous and inhomogeneous elastic half-spaces are a wellknown and prominent feature of wave theory. Abd-Alla and Abo-Dahab [1] investigated time-harmonic sources in a generalized magneto-thermo-viscoelastic continuum with and without energy dissipation. Abd-Alla [2] studied the effect of initial stress and orthotropy on the propagation waves in a hollow cylinder. Abd-Alla et al. [3] presented Rayleigh waves in a magnetoelastic half-space of orthotropic material under an influence of initial stress and gravity field. Garg [4] considered effect of initial stress on harmonic plane homogeneous waves in viscoelastic anisotropic media, Vecsey, et al. [5] investigated shear wave splitting as a diagnostic of variable anisotropic structure of the upper mantle beneath central Fennoscandia. Singh [6], studied wave propagation in a generalized thermoelastic material with voids. Willis and Movchan [7] discussed propagation of elastic energy in a general anisotropic medium. Zhang and Batra [8] wave propagation in functionally graded materials by modified smoothed particle hydrodynamics (MSPH) method. Zhu and Shi [9] discussed wave propagation in non-homogeneous magneto-electro-elastic hollow cylinders. Jiangong, et al. [10] studied wave propagation in non-homogeneous magneto-

*Address correspondence to this author at the Mathematics Department, J.T.C., King Abdul Aziz University, K.S.A., Suadia Arab;

E-mail: srhassan@kau.edu.sa

"Permanent address: Mathematics Department, Faculty of Science, University of Sohag, 82524, Egypt. electro-elastic plates. Bin et al. [11] discussed asymmetric wave propagation in a transversely isotropic half-space in displacement potentials. Khojasteh et al. [12] studied diffraction-biased shear wave fields generated with longitudinal magnetic resonance elastography drivers. Crampin and Peacock [13] investigated shear horizontal waves in transversely inhomogeneous plates. Rayleigh waves in a magnetoelastic initially stressed conducting medium with the gravity field are investigated by El-Naggare et al. [14].

In this paper, the effect of initial stress, magnetic field, non-homogeneity of the medium and gravity field on the propagation of shear wave in an orthotropic elastic solid medium has been discussed using the wave equations which satisfied by the displacement potentials $\varphi$. The frequency equation that determines the velocity of the shear wave has been obtained. The dispersion equations have been obtained, and investigated for different cases. In fact, these equations are in agreement with the corresponding classical results when the medium is isotropic.

\section{FORMULATION OF THE PROBLEM}

Most materials behave as incompressible media and the velocities of longitudinal waves in them are very high. The varieties of hard rocks present in the earth are also almost incompressible. Due to the factors like external pressure, slow process of creep, difference in temperature, manufacturing processes, nitriding, pointing etc., the medium stay under high stresses. These stresses are regarded as initial stresses. Owing to the variation of elastic properties and the presence of these initial stresses, the medium becomes isotropic as well. We consider an unbounded incompressible anisotropic medium under initial stresses $S_{11}$ and $s_{22}$ along the $x, y$ directions, respectively. When the medium is slightly disturbed $(u, v)$, the incremental stresses $s_{11}, s_{12}$ and $s_{22}$ are developed, and the equations of motion in the incremental state become [14]. 


$$
\begin{aligned}
& \frac{\partial \mathrm{s}_{11}}{\partial \mathrm{x}}+\frac{\partial \mathrm{s}_{12}}{\partial \mathrm{x}}-\mathrm{P} \frac{\partial \omega}{\partial \mathrm{y}}-\rho \mathrm{g} \frac{\partial \mathrm{v}}{\partial \mathrm{x}}=\rho \frac{\partial^{2} \mathrm{u}}{\partial \mathrm{t}^{2}} \\
& \frac{\partial \mathrm{s}_{12}}{\partial \mathrm{x}}+\frac{\partial \mathrm{s}_{22}}{\partial \mathrm{y}}-\mathrm{P} \frac{\partial \omega}{\partial \mathrm{x}}+\rho \mathrm{g} \frac{\partial \mathrm{u}}{\partial \mathrm{x}}+\mu_{\mathrm{e}} \mathrm{H}_{0}^{2}\left(\frac{\partial^{2} \mathrm{v}}{\partial \mathrm{x}^{2}}-\frac{\partial^{2} \mathrm{u}}{\partial \mathrm{x} \partial \mathrm{y}}\right)=\rho \frac{\partial^{2} \mathrm{v}}{\partial \mathrm{t}^{2}}
\end{aligned}
$$

where $\mu_{e}$ is the magnetic permeability and $H_{0}$, the intensity of the uniform magnetic field, parallel to $x$-axes, also, $s_{i j}$ is incremental stresses, $(u, v)$ is an incremental deformation, $\omega$ is the rotational component about $z$-axis, $g$ is the acceleration due to gravity The incremental stress- strain relation for an incompressible medium may be taken as [14]

$s_{11}=2 N e_{x x}+s, s_{22}=2 N e_{y y}+s$, and

$\mathrm{s}_{12}=2 \mathrm{Qe}_{\mathrm{xy}}$

$s_{33}=s_{13}=s_{23}$, since the problem is treated in two dimensions $(x, y)$ where $\mathrm{s}=\frac{\mathrm{s}_{11}+s_{22}}{2}, e_{i j}$ is an incremental strain component, and $N$ and $Q$ are the rigidities of the medium. The incompressibility condition $e_{x x}+e_{y y}=0$ is satisfied by.

$\mathrm{u}=-\frac{\partial \phi}{\partial \mathrm{y}}, v=\frac{\partial \phi}{\partial \mathrm{x}}$

Substituting from eqs. (3) and (4). in eqs.(1) and (2) we get

$\frac{\partial \mathrm{s}}{\partial \mathrm{x}}-2 \mathrm{~N} \frac{\partial^{3} \varphi}{\partial \mathrm{x}^{2} \partial \mathrm{y}}+\frac{\partial}{\partial \mathrm{y}}\left[\mathrm{Q}\left(\frac{\partial^{2} \varphi}{\partial \mathrm{x}^{2}}-\frac{\partial^{2} \varphi}{\partial \mathrm{y}^{2}}\right)\right]-\frac{\mathrm{P}}{2}\left(\frac{\partial^{3} \varphi}{\partial \mathrm{x}^{2} \partial \mathrm{y}}+\frac{\partial^{3} \varphi}{\partial \mathrm{y}^{3}}\right)-$

$-\rho \mathrm{g} \frac{\partial^{2} \varphi}{\partial \mathrm{x}^{2}}=-\rho\left[\frac{\partial^{3} \varphi}{\partial \mathrm{t}^{2} \partial \mathrm{y}}\right]$

$\frac{\partial s}{\partial y}+Q\left[\frac{\partial^{3} \phi}{\partial x^{3}}-\frac{\partial^{3} \phi}{\partial x \partial y^{2}}\right]+\frac{\partial}{\partial y}\left[2 N \frac{\partial^{2} \phi}{\partial x \partial y}\right]-\frac{P}{2}\left(\frac{\partial^{3} \phi}{\partial x^{3}}+\frac{\partial^{3} \phi}{\partial x \partial y^{2}}\right)-\rho g \frac{\partial^{2} \phi}{\partial x \partial y}+$ $+\mu_{e} H_{0}^{2}\left[\frac{\partial^{3} \phi}{\partial x^{3}}+\frac{\partial^{3} \phi}{\partial x \partial y^{2}}\right]=\rho\left[\frac{\partial^{3} \phi}{\partial t^{2} \partial x}\right]$

Assuming non-homogeneities as

$Q=Q_{0}(1+a y)$

$N=N_{0}(1+b y)$

$\rho=\rho_{0}(1+c y)$

Substituting from eq. (7) in eqs. (5) and (6) we get

$$
\begin{gathered}
\frac{\partial^{2} s}{\partial x \partial y}-\left[2 N_{0} b-2 a Q_{0}+\rho_{0} g(1+c y)\right] \frac{\partial^{3} \varphi}{\partial x^{2} \partial y}-\left[2 N_{0}(1+b y)-Q_{0}(1+a y)+\frac{P}{2}\right] \\
\frac{\partial^{4} \varphi}{\partial \mathrm{x}^{2} \partial \mathrm{y}^{2}}-2 a Q_{0} \frac{\partial^{3} \varphi}{\partial y^{3}}-\left[Q_{0}(1+a y)+\frac{P}{2}\right] \frac{\partial^{4} \varphi}{\partial y^{4}}-\rho_{0} c g \frac{\partial^{2} \varphi}{\partial x^{2}}= \\
=-\rho_{0}(1+c y) \frac{\partial^{4} \varphi}{\partial t^{2} \partial y^{2}}-\rho_{0} c \frac{\partial^{3} \varphi}{\partial t^{2} \partial y}
\end{gathered}
$$

$$
\begin{aligned}
& \frac{\partial^{2} s}{\partial x \partial y}+\left[Q_{0}(1+a y)-\frac{P}{2}+\mu_{e} H_{0}^{2}\right] \frac{\partial^{4} \phi}{\partial x^{4}}+\left[2 N_{0} b-\rho_{0} g(1+c y)\right] \frac{\partial^{3} \phi}{\partial x^{2} \partial y}+ \\
& {\left[2 N_{0}(1+b y)-Q_{0}(1+a y)-\frac{P}{2}+\mu_{e} H_{0}^{2}\right] \frac{\partial^{4} \phi}{\partial x^{2} \partial y^{2}}=\rho_{0}(1+c y) \frac{\partial^{4} \phi}{\partial t^{2} \partial x^{2}}}
\end{aligned}
$$

Eliminating $s$ from eqs. (8) and (9), we get

$$
\begin{aligned}
& {\left[Q_{0}(1+a y)-\frac{P}{2}+\mu_{e} H_{0}^{2}\right] \frac{\partial^{4} \phi}{\partial x^{4}}+\left[4 N_{0}(1+b y)-2 Q_{0}(1+a y)-\frac{P}{2}+\mu_{e} H_{0}^{2}\right]} \\
& \frac{\partial^{4} \phi}{\partial x^{2} \partial y^{2}}+\left[Q_{0}(1+a y)+\frac{P}{2}\right] \frac{\partial^{4} \phi}{\partial y^{4}}+2 a Q_{0} \frac{\partial^{3} \phi}{\partial y^{3}}-\left[2 a Q_{0}-4 N_{0} b\right] \frac{\partial^{3} \phi}{\partial x^{2} \partial y}+ \\
& +\rho_{0} c g \frac{\partial^{2} \phi}{\partial x^{2}}=\rho_{0}(1+c y)\left[\frac{\partial^{4} \phi}{\partial t^{2} \partial x^{2}}+\frac{\partial^{4} \phi}{\partial t^{2} \partial y^{2}}\right]+\rho_{0} c \frac{\partial^{3} \phi}{\partial t^{2} \partial y}
\end{aligned}
$$

\section{SOLUTION OF THE PROBLEM}

For propagation of sinusoidal waves in any arbitrary direction, we take the solution of eq. (10) as

$\phi(x, y, t)=A e^{i k\left(x \cos \theta+y \sin \theta-c_{1} t\right)}$

where $\theta$ is the angle made by the direction of propagation with the $x$-axes, and $c_{1}$ and $k$ are the velocity of propagation and wave number, respectively.

Using eq. (11) in eq. (10) and equating real and imaginary parts separately, we get

$$
\begin{gathered}
\left(\frac{c_{1}}{\beta}\right)^{2}=\frac{1}{1+c y}\left\{\begin{array}{l}
{\left[(1+a y)-\frac{P}{2 Q_{0}}+\frac{\mu_{e} H_{0}^{2}}{Q_{0}}\right] \cos ^{4} \theta+} \\
\left.+4 \frac{N_{0}}{Q_{0}}(1+b y)-2(1+a y)+\frac{\mu_{e} H_{0}^{2}}{Q_{0}}\right] \\
\cos ^{2} \theta \sin ^{2} \theta+\left[(1+a y)+\frac{P}{2 Q_{0}}\right] \sin ^{4} \theta-\frac{c g}{k^{2} \beta^{2}} \cos ^{2} \theta
\end{array}\right\} \\
\left(\frac{c_{1}}{\beta}\right)^{2}=\left[4 \frac{N_{0}}{Q_{0}} \frac{b}{c}-\frac{2 a}{c}\right] \cos ^{2} \theta+2 \frac{a}{c} \sin ^{2} \theta
\end{gathered}
$$

\section{PARTICULAR CASES}

In order to gain more insight information the following cases have been discussed.

Analysis of eq. (12) obtained by equating the real part of equations of motion:

\section{Case I}

In this case $Q$ is homogeneous $(\mathrm{a} \rightarrow 0)$ i.e., rigidity along vertical direction is constant

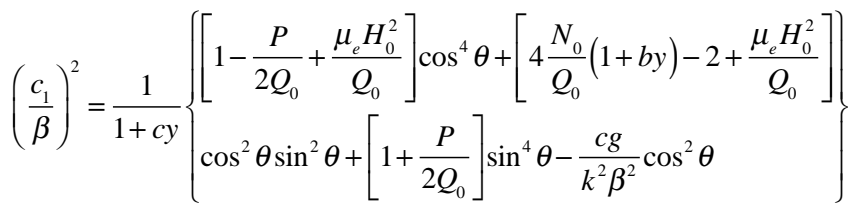


The velocity of a long $x$-direction $\left(\cos \theta=1, \sin \theta=0, c_{1}=c_{11}\right)$ as

$$
\begin{aligned}
& \left(\frac{c_{11}}{\beta}\right)^{2}=\frac{1}{1+c y}\left\{\left[1-\frac{P}{2 Q_{0}}+\frac{\mu_{e} H_{0}^{2}}{Q_{0}}\right]-\frac{c g}{k^{2} \beta^{2}}\right\} \\
& c_{11}^{2}=\frac{1}{1+c y}\left\{\beta^{2}\left[1-\frac{P}{2 Q_{0}}+\frac{\mu_{e} H_{0}^{2}}{Q_{0}}\right]-\frac{c g}{k^{2}}\right\}
\end{aligned}
$$

which depends on the initial stress, gravity field and magnetic field. Similarly the velocity of propagation along $y$-direction $\left(\cos \theta=0, \sin \theta=1, c_{1}=c_{22}\right)$, is obtained as

$$
c_{22}^{2}=\frac{\beta^{2}}{1+c y}\left[1+\frac{P}{2 Q_{0}}\right]
$$

\section{Case II}

In this case $N$ is homogeneous ( $b \rightarrow 0$ ) i.e., rigidity along horizontal direction is constant

$$
\left(\frac{c_{1}}{\beta}\right)^{2}=\frac{1}{1+c y}\left\{\begin{array}{l}
{\left[(1+a y)-\frac{P}{2 Q_{0}}+\frac{\mu_{e} H_{0}^{2}}{Q_{0}}\right] \cos ^{4} \theta+} \\
+\left[4 \frac{N_{0}}{Q_{0}}-2(1+a y)+\frac{\mu_{e} H_{0}^{2}}{Q_{0}}\right] \cos ^{2} \theta \sin ^{2} \theta \\
+\left[(1+a y)+\frac{P}{2 Q_{0}}\right] \sin ^{4} \theta-\frac{c g}{k^{2} \beta^{2}} \cos ^{2} \theta
\end{array}\right\}
$$

The velocity of a long $x$-direction $(\cos \theta=1, \sin \theta=0$, $c_{1}=c_{11}$ ) is given by

$c_{11}^{2}=\frac{1}{1+c y}\left\{\beta^{2}\left[(1+a y)-\frac{P}{2 Q_{0}}+\frac{\mu_{e} H_{0}^{2}}{Q_{0}}\right]-\frac{c g}{k^{2}}\right\}$

which depends on depth $y$, gravity field and magnetic field. The velocity of propagation along $y$-direction $\left(\cos \theta=0, \sin \theta=1, c_{1}=c_{22}\right)$, is given by

$c_{22}^{2}=\frac{\beta^{2}}{1+c y}\left\{\left[(1+a y)+\frac{P}{2 Q_{0}}\right]\right\}$

For $P>0$, the velocity along $y$-direction may increase considerably at a distance from free surface and the wave becomes dispersive.

\section{Case III}

In this case $N, Q$ and $\rho$ are homogeneous $(a \rightarrow 0, b \rightarrow 0, c \rightarrow 0)$

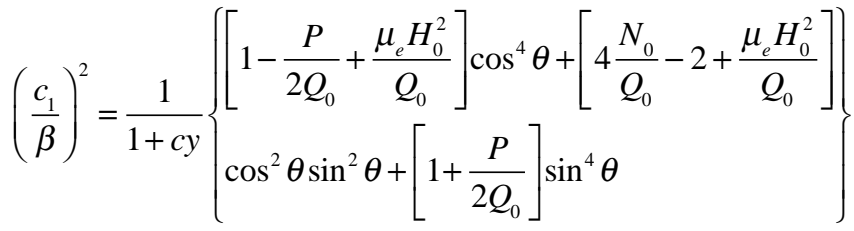

In the absence of initial stress the velocity equation becomes

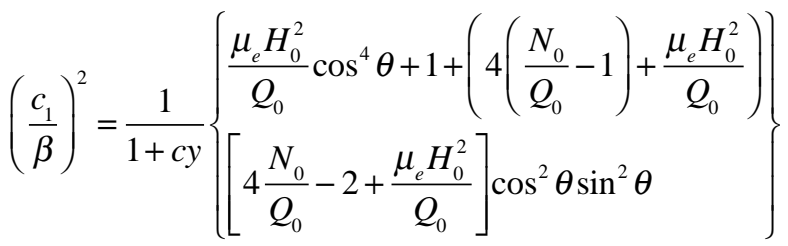

In $x$-direction $\left(\cos \theta=1, \sin \theta=0, c_{1}=c_{11}\right)$ the velocity is given by

$c_{11}^{2}=\beta^{2}\left[1+\frac{\mu_{e} H_{0}^{2}}{Q_{0}}\right]$

and in $y$-direction $\left(\cos \theta=0, \sin \theta=1, c_{1}=c_{22}\right)$ the velocity is given by

$c_{22}^{2}=\beta^{2}$

\section{Case IV}

In absence of initial stress $P \rightarrow 0$, the velocity is obtained as:

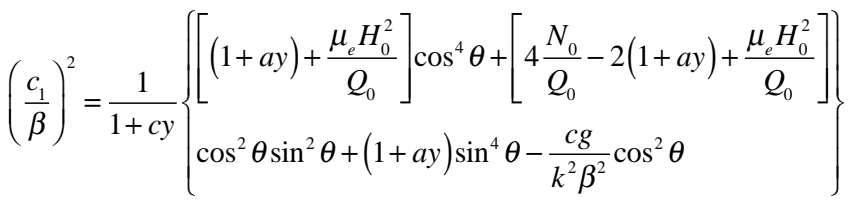

The velocity of a long $x$-direction $(\cos \theta=1, \sin \theta=0$, $\left.c_{1}=c_{11}\right)$ is given by

$\left(\frac{c_{1}}{\beta}\right)^{2}=\frac{1}{1+c y}\left[(1+a y)+\frac{\mu_{e} H_{0}^{2}}{Q_{0}}-\frac{c g}{k^{2} \beta^{2}}\right]$

The velocity of a long $y$-direction $(\cos \theta=0$, $\left.\sin \theta=1, \quad c_{1}=c_{22}\right)$ is given by

$\left(\frac{c_{1}}{\beta}\right)^{2}=\frac{1+a y}{1+c y}$

\section{Case V}

In this case, the magnetic field is neglected $\left(H_{0} \rightarrow 0\right)$, the velocity equation is given by

$\left(\frac{c_{1}}{\beta}\right)^{2}=\frac{1}{1+c y}\left\{\begin{array}{l}{\left[(1+a y)-\frac{P}{2 Q_{0}}\right] \cos ^{4} \theta+\left[4 \frac{N_{0}}{Q_{0}}-2(1+a y)\right] \cos ^{2} \theta \sin ^{2} \theta+} \\ +\left[(1+a y)+\frac{P}{2 Q_{0}}\right] \sin ^{4} \theta-\frac{c g}{k^{2} \beta^{2}} \cos ^{2} \theta\end{array}\right\}$

The velocity of a long $x$-direction $(\cos \theta=1, \sin \theta=0$, $\left.c_{1}=c_{11}\right)$ is given by 
$\left(\frac{c_{11}}{\beta}\right)^{2}=\frac{1}{1+c y}\left\{\left[(1+a y)-\frac{P}{2 Q_{0}}-\frac{c g}{k^{2} \beta^{2}}\right]\right\}$

The velocity of a long $y$-direction $(\cos \theta=0, \sin \theta=1$, $c_{1}=c_{22}$ ) is given by

$\left(\frac{c_{22}}{\beta}\right)^{2}=\frac{1}{1+c y}\left\{\left[(1+a y)-\frac{P}{2 Q_{0}}\right]\right\}$

Analysis of eq. (13) obtained by equating imaginary parts of equation of motion.

In absence the initial stress $P$ in equation (13), following three cases have been analyzed.

\section{Case I}

In this case $Q$ is homogeneous $(a \rightarrow 0)$ i.e., rigidity along vertical direction is constant

$$
\left(\frac{c_{1}}{\beta}\right)^{2}=2\left(\left[\frac{N_{0}}{Q_{0}} \frac{b}{c}\right] \cos ^{2} \theta\right)
$$

This shows that velocity of shear wave is always damped. The velocity of wave along $x$-direction $(\cos \theta=1$, $\left.\sin \theta=0, c_{1}=c_{11}\right)$ is obtained as

$\left(\frac{c_{11}}{\beta}\right)^{2}=2\left(\left[\frac{2 N_{0}}{Q_{0}} \frac{b}{c}\right]\right)$

This shows that actual velocity in $x$-direction is damped by $\left(\frac{2 N_{0} b}{Q_{0} c}\right)$, and no damping takes place along $y$-direction.

\section{Case II}

In this case $N$ is homogeneous $(b \rightarrow 0)$, i.e., rigidity along horizontal direction is constant.

$\left(\frac{c_{1}}{\beta}\right)^{2}=2\left(\left[-\frac{a}{c}\right] \cos ^{2} \theta+2\left[\frac{a}{c}\right] \sin ^{2} \theta\right)$

The velocity of wave along $x$-direction $(\cos \theta=1$, $\left.\sin \theta=0, c_{1}=c_{11}\right)$ is given by

$\left(\frac{c_{11}}{\beta}\right)^{2}=2\left[-\frac{a}{c}\right]$

The existence of negative sign shows that damping does not take place along $x$-direction for $(b \rightarrow 0)$. The velocity along $y$-direction is given by

$\left(\frac{c_{22}}{\beta}\right)^{2}=2\left[\frac{a}{c}\right]$

Indicating that a damping of magnitude $\left(\frac{2 a}{c}\right)$ takes place along $y$-direction .

\section{Case III}

In this case $N$ and $Q$ are homogeneous but density is linearly varying with depth:

$\left(\frac{c_{1}}{\beta}\right)^{2}=0$

i.e., no damping takes place. Rather than perform the tedious analysis required in obtaining higher-order approximations in the manner outlined above.

\section{NUMERICAL RESULTS AND DISCUSSION}

We wish to investigate the variation of shear waves velocity in a perfectly conducting medium under effect of magnetic field, initial stress and gravity field. To get numerical information on the velocity of shear wave in the nonhomogenous initially stressed medium we introduce the following non-dimensional parameters:

$\bar{a}=\frac{a}{b}, \bar{b}=b y, \bar{c}=\frac{c}{b}, \bar{c}_{1}=\frac{c_{1}}{b}, \bar{N}_{0}=\frac{N_{0}}{Q_{0}}$,

$\bar{P}=\frac{P_{0}}{2 Q_{0}}, \bar{g}=\frac{g}{\rho c^{2}}, \bar{H}=\frac{H}{2 Q_{0}}$

Using these parameters in the Eq. (12), we obtain:

$\bar{c}_{1}^{2}=\frac{1}{(1+\bar{c} \bar{b})}\left\{\begin{array}{l}{[1+\bar{c} \bar{b}-\bar{P}+\bar{H}] \cos ^{4} \theta+2[\bar{N}(1+\bar{b})-(1+\bar{a} \bar{b})+\bar{H}]} \\ \cos ^{2} \theta \sin ^{2} \theta+[1+\bar{a} \bar{b}+\bar{P}] \sin ^{4} \theta-\overline{c g} \cos ^{2} \theta\end{array}\right\}$

Figs. (1-5) show the effect of a non-homogeneous anisotropic incompressible, magnetic field, gravity field and initially stressed respectively on shear wave velocity $c$ with respect to depth $b$, it is obvious that shear wave velocity increases with the increasing of the depth $b$, also it is increases with the increasing of the gravity field, magnetic field and it decreases with an increase in of the initial stress. While, Figs. (6-8) with respect to angle $\theta$, it is obvious that shear wave velocity vibrations with the increasing of the angle $\theta$.

Fig. (1) shows the variation in velocities of shear wave in the direction of $\theta=30$ with $x$-axis at different depth and different values of density parameter:

$a=4 ; c=0.7,0.8,0.9 ; P=0.5$;

$g=.1 \mathrm{~cm} / \mathrm{sec}^{2} N=2.5$ and $H=0.3$

The velocity of the shear-wave velocity increases as depth increases. Fig. (2) shows the variation in velocities of shear wave in the direction of $\theta=30$ with $x$-axis at different depth and different values of rigidities parameter:

$a=3,3.5,4 ; \quad c=0.8 ; P=0.5 ; g=.1 \mathrm{~cm} / \mathrm{sec}^{2}$

$N=2.5$ and $H=0.3$. The velocity of the shear-wave increases as depth increases. Fig. (3) shows the variation in velocities of shear wave in the direction of $\theta=30$ with $x$ axis at different depth and different values of $N$ (anisotropy): 


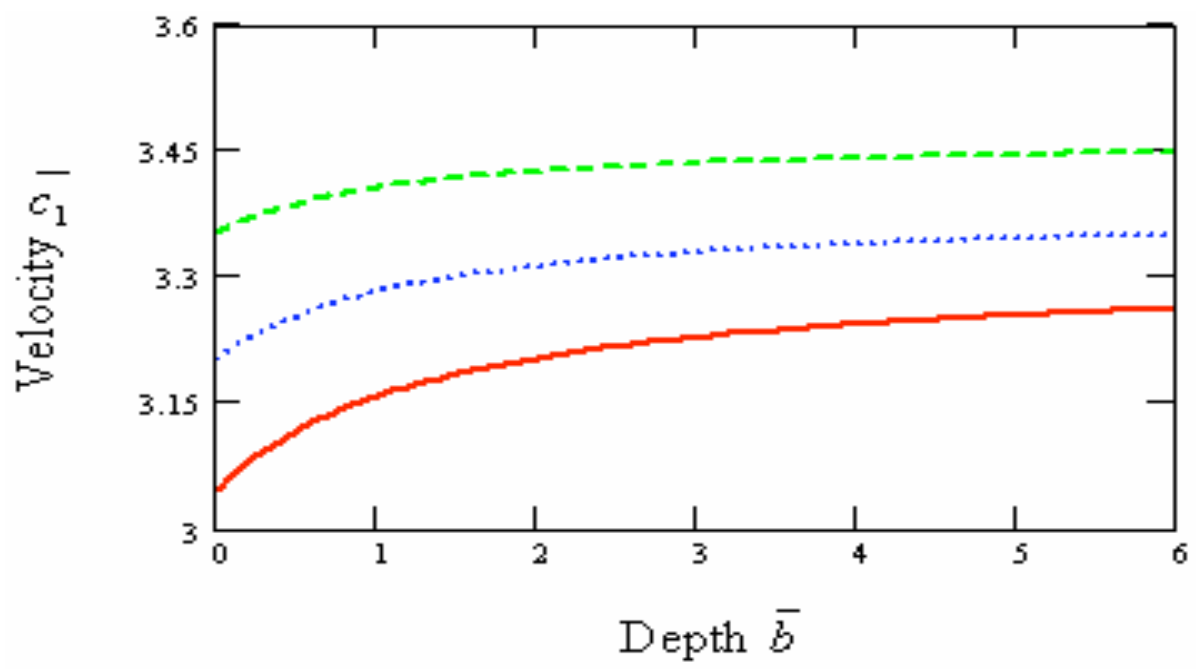

Fig. (1). Variation in velocities of shear wave in the direction of $\theta=30^{\circ}$ with x-axis at different depth and different values of density parameter: $\bar{a}=4 ; \bar{c}=0.7,0.8,0.9 ; \bar{P}=0.5 ; \bar{g}=0.1 ; \bar{N}=2.5$ and $\bar{H}=0.3$.

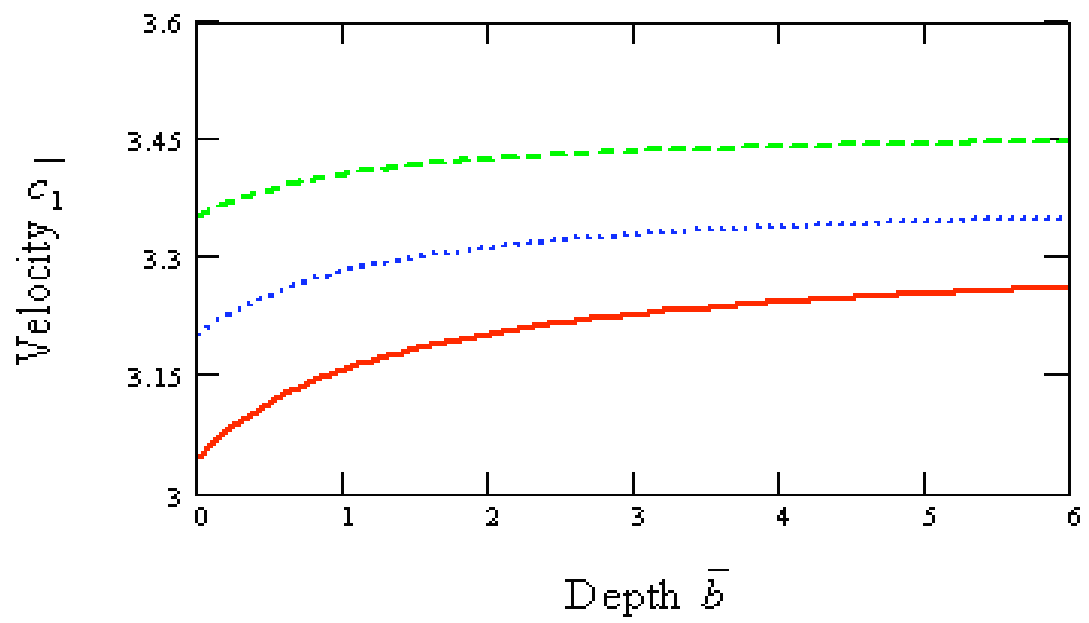

Fig. (2). Variation in velocities of shear wave in the direction of $\theta=30^{\circ}$ with x-axis at different depth and different values of rigidities parameter: $\bar{a}=3,3.5,4 ; \bar{c}=0.8 ; \bar{P}=0.5 ; \bar{g}=0.1 ; \bar{N}=2.5$ and $\bar{H}=0.3$.

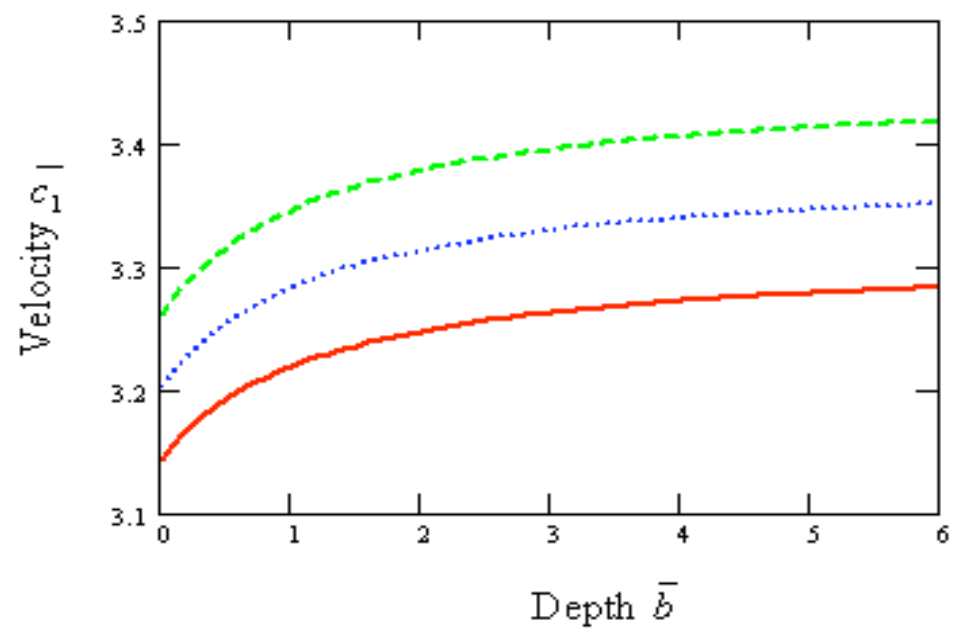

Fig. (3). Variation in velocities of shear wave in the direction of $\theta=30^{\circ}$ with x-axis at different depth and different values of $\bar{N}$. (anisotropy): $\bar{N}=2,2.5,3 ; \bar{a}=4 ; \bar{c}=0.8 ; \bar{P}=0.5 ; \bar{g}=0.1 ;$ and $\bar{H}=0.3$. 


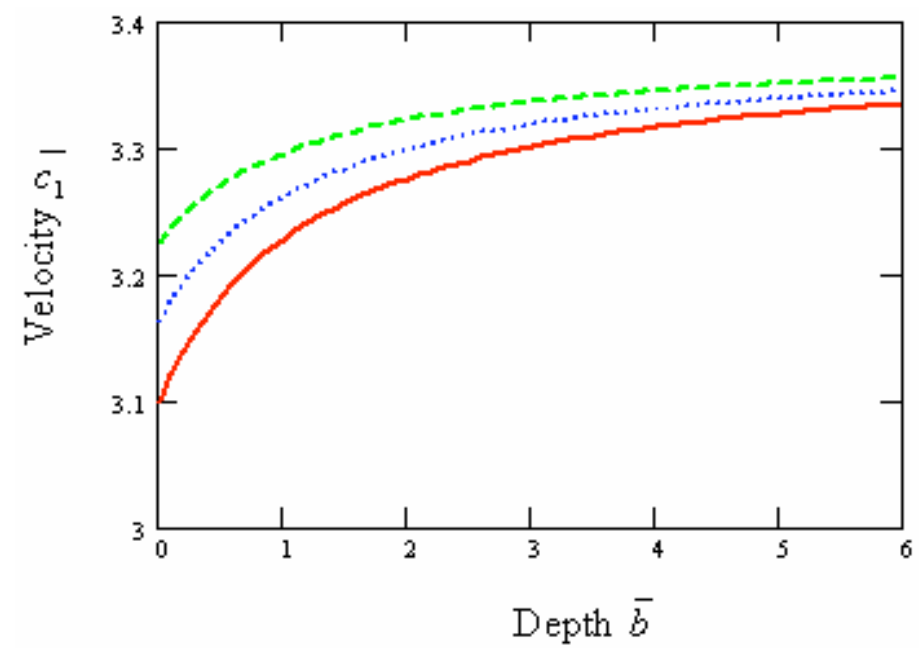

Fig. (4). Variation in velocities of shear wave in the direction of $\theta=30^{\circ}$ with x-axis at different depth and different values initial stress parameter $\bar{P}$. when $\bar{P}=-0.8,0.0,0.8 ; \bar{c}=0.8 ; \bar{g}=0.1 ; \bar{a}=4 ; \bar{N}=2.5$ and $\bar{H}=0.3$.

$N=2,2.5,3 ; a=4 ; c=0.8 ; P=0.5 ; g=.1 \mathrm{~cm} / \mathrm{sec}^{2}$ and

$H=0.3$. Also, Fig. (3) gives the information of variation of velocity for different values of anisotropic factor and reflects that with the increase in the values of $N$, the velocity of shear wave increases. Fig. (4) shows the variation in velocities of shear wave in the direction of $\theta=30$ with $x$-axis at different depth and different values initial stress parameter $P$ when

$P=-0.8,0.0,0.8 ; c=0.8 ; g=.1 \mathrm{~cm} / \mathrm{sec}^{2} ; a=4 ; N=2.5$

and $H=0.3$. The velocity of the wave increases as depth increases. Fig. (5) shows the variation in velocities of shear wave in the direction of $\theta=30$ with $x$-axis at different depth and different values of initial stress medium with
The velocity of the wave increases as depth increases. Figs. $(\mathbf{6}, \mathbf{8})$ show the variation of shear-wave velocity with $x$-axis at different values of angles $\theta$ and different values of anisotropy factor and it increases with increasing magnetic field $H$. Fig. (7) shows the variation of shear-wave velocity with $x$-axis at different values of angles $\theta$ and it decreases with an increase of initial stress $P$.

Figs. (1-8) show the variation of the shear wave velocity with respect to initial stress. The shear wave velocity is inversely proportional to the depth; also, the increment in both shear wave velocity and initial stress is proportional to the magnetic field and gravity field. Lastly, from Figs. (1-8) it is clear the velocity of shear wave is decrease by increasing of the initial stress, while it is increased by the magnetic field.

$a=4 ; c=0.8 ; P=0.5 ; g=.1 \mathrm{~cm} / \sec ^{2} N=2.5$ and

$H=0.1,0.5,0.9$.

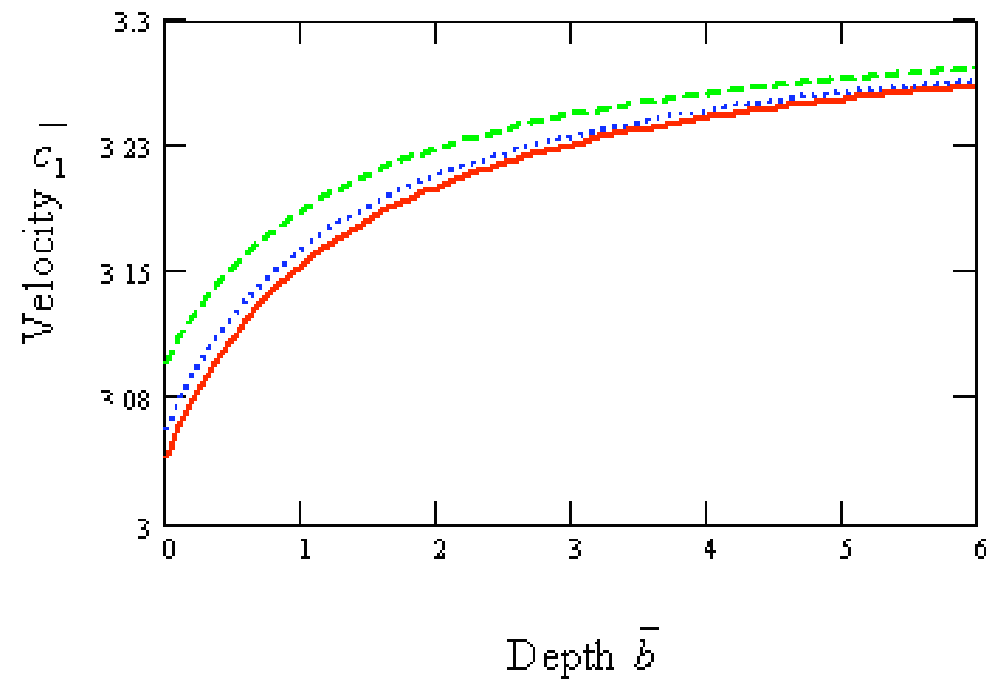

Fig. (5). Variation in velocities of shear wave in the direction of $\theta=30^{\circ}$ with $\mathrm{x}$-axis at different depth and different values initial stress medium with $\bar{P} \quad \bar{a}=4 ; \bar{c}=0.8 ; \bar{P}=0.5 ; \bar{g}=0.1 ; \bar{N}=2.5$ and $\bar{H}=0.1,0.5,0.9$. 


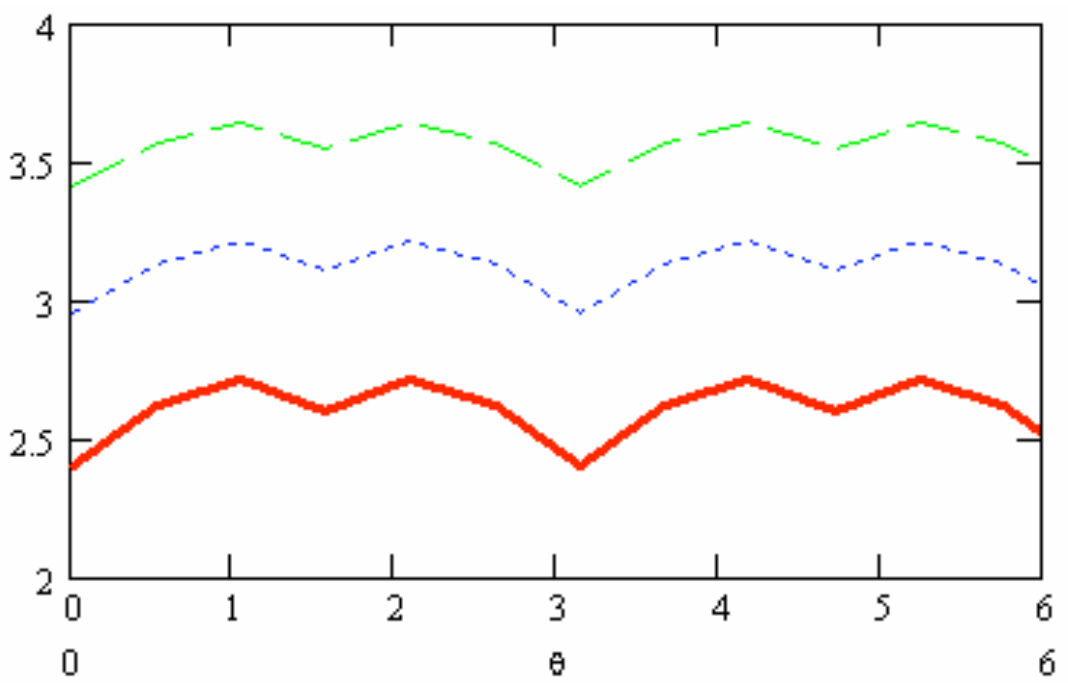

Fig. (6). Variation of velocities of shear wave with respect to $\theta$ with x-axis,

$\bar{P}=0.5 ; \bar{H}=0.3 ; \bar{g}=0.1 ; \bar{a}=3 ; \bar{N}=2.5 ; \bar{b}=0.2$ and $\bar{c}=0.5,0.8,1.1$.

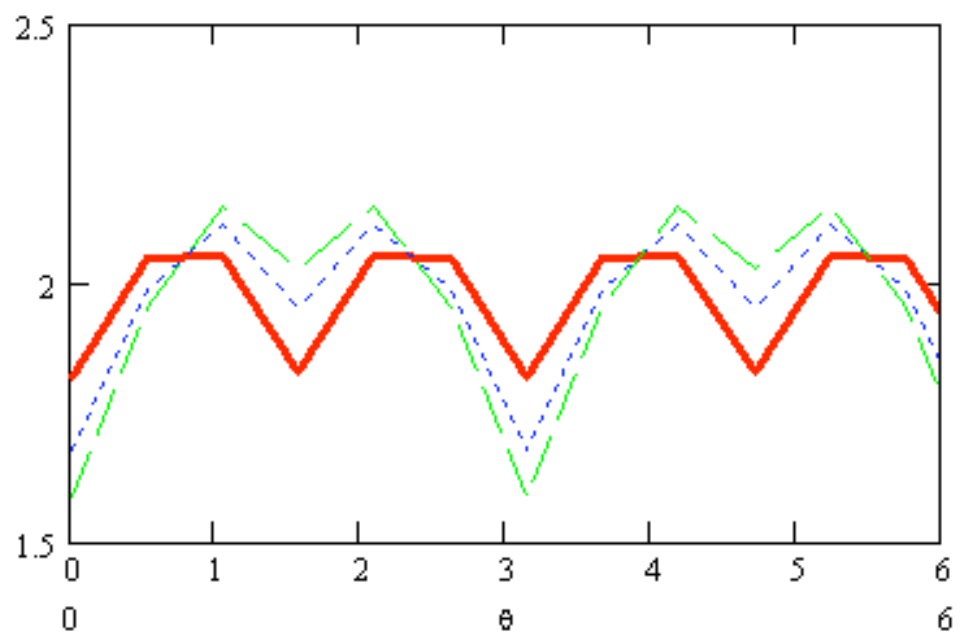

Fig. (7). Variation of velocities of shear wave with respect to $\theta$ with $\mathrm{x}$-axis,

$\bar{P}=0.2,0.5,0.8 ; \bar{H}=0.3 ; \bar{g}=0.1 ; a=3 ; \bar{N}=2.5 ; \bar{b}=0.2$ and $\bar{c}=0.8$.

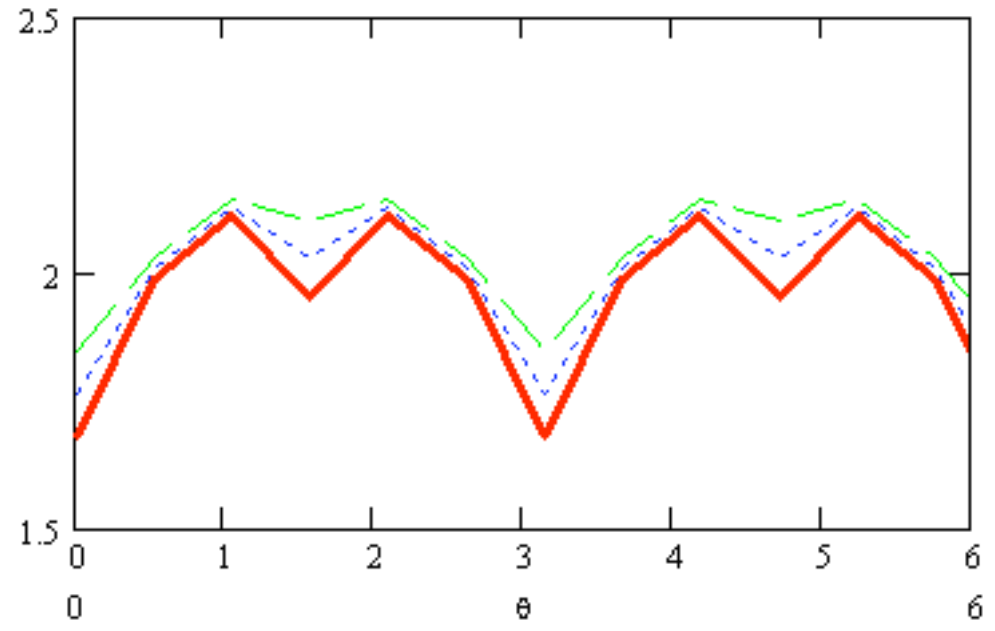

Fig. (8). Variation of velocities of shear wave with respect to $\theta$ with $\mathrm{x}$-axis,

$\bar{c}=0.8 ; \bar{P}=0.5 ; \bar{g}=0.1 ; \bar{a}=3 ; \bar{N}=2.5 ; \bar{b}=0.2$ and $\bar{H}=0.3,0.6,0.8$. 


\section{CONCLUSIONS}

The anisotropy, magnetic field, gravity field, nonhomogeneity of the medium, the initial stress, the direction of propagation and the depth have considerable effect in the velocity of propagation of shear wave and attracts the attention of earth scientists in their work. Numerical computation shows that the presence of initial compressive stress in the medium, magnetic field and gravity field, reduce the velocity of propagation while the tensile stress increases it. It is found that the variation in parameters associated with anisotropy and non-homogeneity of the medium directly affects the velocity of the wave. The velocity of propagation also depends on the inclination of the direction of propagation; an increase in the inclination angle decreases the velocity in the beginning, takes a minimum value before increasing.

\section{REFERENCES}

[1] Abd-Alla AM, Abo-Dahab SM. Time-harmonic sources in a generalized magneto-thermo-viscoelastic continuum with and without energy dissipation. Appl Math Model 2009; 33(5): 2388-402.

[2] Abd-Alla AM. The effect of initial stress and orthotropy on the propagation waves in a hollow cylinder. Appl Math Comput 1999; 106(3): 237-44

[3] Abd-Alla AM, Hammad AH, Abo-Dahab SM. Rayleigh waves in a magneto elastic half-space of orthotropic material under influence of initial stress and gravity field. Appl Math Comput 2004; 154(2): 583-97.
[4] Neetu G. Effect of initial stress on harmonic plane homogeneous waves in viscoelastic anisotropic media. J Sound Vib 2007; 303(3-5): 515-25.

[5] Vecsey L, Plomerov J, Kozlovskaya E, Babuka V. Shear wave splitting as a diagnostic of variable anisotropic structure of the upper mantle beneath central Fennoscandia. Tectonophysics 2007; 438(1): 57-77.

[6] Baljeet S. Wave propagation in a generalized thermoelastic material with voids. Appl Math Comput 2007; 189(1): 698-709.

[7] Willis JR, Movchan NV. Propagation of elastic energy in a general anisotropic medium. J Sound Vib 2007; 302(4-5): 629-42.

[8] Zhang GM, Batra RC. Wave propagation in functionally graded materials by modified smoothed particle hydrodynamics (MSPH) method. J Comput Phys 2007; 222(1): 374-390.

[9] Zhu BJ, Shi YL. Wave propagation in non-homogeneous magnetoelectro-elastic hollow cylinders. Ultrasonic 2008; 48(8): 664-77.

[10] Jiangong Yu, Qiujuan Ma, Shan Su. Wave propagation in nonhomogeneous magneto-electro-elastic plates. J Sound Vib 2008; 317(2): 250-64.

[11] Wu Bin, Jiangong Yu, Cunfu He. Asymmetric wave propagation in a transversely isotropic half-space in displacement potentials. Int $\mathbf{J}$ Eng Sci 2008; 46(7): 690-710.

[12] Khojasteh A, Rahimian M, Eskandari M, Pak YS. Diffractionbiased shear wave fields generated with longitudinal magnetic resonance elastography drivers. Magn Reson Imaging 2008; 26(6): 770-80.

[13] Stuart C, Sheila P. Shear horizontal waves in transversely inhomogeneous plates. Wave Motion 2008; 45(5): 605-15.

[14] El-Naggar AM, Abda-Alla AM, Ahmad SM. Rayliegh waves in amagnetoelastic intially stressed conducting medium with the gravity field. Bull Calcutta Math Soc 1994; 86: 51-6.

(C) Abd-Alla et al.; Licensee Bentham Open.

This is an open access article licensed under the terms of the Creative Commons Attribution Non-Commercial License (http://creativecommons.org/licenses/by-nc/3.0/) which permits unrestricted, non-commercial use, distribution and reproduction in any medium, provided the work is properly cited. 